Check for updates

Cite this: Phys. Chem. Chem. Phys., 2018, 20, 10155

Received 21st December 2017 Accepted 21st March 2018

DOI: $10.1039 / c 7 c p 08555 a$

rsc.li/pccp

\section{Experimental evidence of TICT state in 4-piperidinyl-1,8-naphthalimide - a kinetic and mechanistic study}

\author{
Zoltán Szakács, (D) a Sylvia Rousseva, ${ }^{a}$ Márton Bojtár, ${ }^{b}$ Dóra Hessz, ${ }^{c}$ István Bitter, ${ }^{b}$ \\ Mihály Kállay, (D) ${ }^{d}$ Michiel Hilbers, ${ }^{e}$ Hong Zhang (iD ${ }^{e}$ and Miklós Kubinyi (D) *ac
}

\begin{abstract}
The excited state processes in $N$-propyl-4-piperidinyl-1,8-naphthalimide have been studied by measuring its fluorescence spectra and decay curves in solvents of different polarity and viscosity and also in a frozen solvent glass. The results unanimously proved the formation of a dark twisted intramolecular charge transfer (TICT) state from the emissive charge transfer (CT) species, the direct product of excitation. The rate coefficients of the TICT process and the deactivations of the CT and TICT species were determined, using a reversible two-state kinetic model. The temperature dependence of the kinetic data was consistent with a kinetic barrier consisting of three terms, the inherent barrier of the reaction, and the contributions of the solute-solvent interactions related to the solvent viscosity and polarity. The potential energy surfaces were calculated in the $\mathrm{S}_{0}$ and the $\mathrm{S}_{1}$ states along the coordinate of turning motion which was conclusive concerning the direction of the twisting and indicated a possible conformational change of the piperidinyl unit. The theoretical calculations confirmed that the TICT species is dark and has a stronger charge transfer character compared to the CT state.
\end{abstract}

\section{Introduction}

1,8-Naphthalimides (NIs) with an electron donor substituent in the 4-position, constitute an important class of push-pull type fluorescent compounds. Their fluorescence wavelengths and in particular, their fluorescence quantum yields are sensitive to the local environment. This attractive feature led to numerous naphthalimide-based fluorescent probes and chemosensors ${ }^{1,2}$ for various cations, ${ }^{3,4}$ anions, ${ }^{5}$ small biomolecules ${ }^{6,7}$ and proteinprotein interactions. ${ }^{8,9}$

The parent compound, 1,8-naphthalimide, and its derivatives with weak donor/acceptor substituents exhibit low fluorescence quantum yields in apolar solvents, somewhat higher values in polar aprotic solvents, which increase further in protic solvents.

\footnotetext{
${ }^{a}$ Department of Physical Chemistry and Materials Science, Budapest University of Technology and Economics, 1521 Budapest, Hungary. E-mail:kubinyi@mail.bme.hu

${ }^{b}$ Department of Organic Chemistry and Technology, Budapest University of Technology and Economics, 1521 Budapest, Hungary

${ }^{c}$ Institute of Materials and Environmental Chemistry, Research Center for Natural Sciences, Hungarian Academy of Sciences, P.O. Box 286, 1519 Budapest, Hungary

${ }^{d}$ MTA-BME Lendület Quantum Chemistry Research Group, Department of Physical Chemistry and Materials Science, Budapest University of Technology and Economics, 1521 Budapest, Hungary

${ }^{e}$ Van't Hoff Institute for Molecular Sciences, University of Amsterdam, P.O. Box 94157, 1090 GD Amsterdam, The Netherlands
}

The effect is due to an efficient intersystem crossing between their $S_{1}$ and $T_{2}$ excited states, lying close in apolar media. The interactions with polar, and in particular, with hydrogen-bond donor solvents shift the $T_{2}$ state to higher, the $S_{1}$ state to lower energies, reducing the effect. ${ }^{10}$

Introducing an electron donor substituent into the 4-position of 1,8-NI, the longest wavelength absorption and fluorescence bands are shifted to longer wavelengths. ${ }^{11-14}$ In most cases, the fluorescence quantum yield becomes higher, because, with the lowering of the energy of the $S_{1}$ state, the $S_{1}-T_{2}$ energy gap becomes larger, reducing the yield of inter-system crossing. ${ }^{11,13}$ The excitation is accompanied by an intramolecular charge transfer (CT) - an electron transfer from the substituent to the electron withdrawing dicarboximide group. ${ }^{15}$ The excited species are stabilized by polar and more efficiently by protic polar solvents, as indicated by the redshift of the fluorescence band in polar media. The photogenerated higher electric field can serve for a fluorescence quenching mechanism which is called generally as photoinduced electron (charge) transfer (PET) in the literature. ${ }^{16}$ This phenomenon is of key importance in the construction of NI-based 'turn-on' fluorescent chemosensors. ${ }^{17}$ Most frequently, they contain an electron rich receptor unit attached through a spacer to their 4-position, or an electron withdrawing receptor unit connected through a spacer to their imide nitrogen atom. Their fluorescence quenched strongly in free state via PET mechanism, is restored when the analyte binds to the receptor unit, shielding its local charge. ${ }^{2}$ 
<smiles>CCCN1C(=O)c2cccc3cccc(c23)C1=O</smiles>

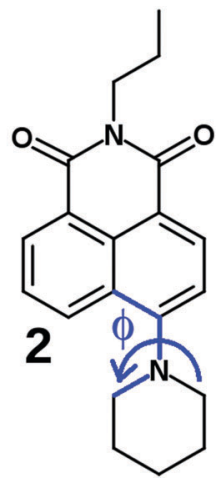

Fig. 1 The investigated compounds 1 and 2, $\Phi$ is the fixed dihedral angle in the computational TICT study.

In NIs with more rigid electron donating groups on their 4carbon atom, a twisted intramolecular charge transfer (TICT) process has also been discovered. ${ }^{18}$ The TICT state may be emissive, manifesting in a dual fluorescence. Examples for this are NIs with a styrene or phenoxy unit inserted between an electron donor group and their 4-position. ${ }^{19,20}$ More frequently, however, the TICT state is dark, leading only to the weakening of the emission from the CT excited state. ${ }^{21}$ The TICT of pushpull NIs is of significance in several potential applications, like when these molecules act as polarity ${ }^{8,9}$ and viscosity probes, ${ }^{22}$ the inhibition of TICT in solid state leads to aggregation induced emission, ${ }^{23-26}$ TICT and $E / Z$ isomerization may occur as simultaneous reactions. ${ }^{19}$

Despite the fluorescence properties of several NIs have been interpreted in terms of two (a CT and a TICT) interconverting $\mathrm{S}_{1}$ excited states, a comprehensive photophysical study had lacked on a more simple push-pull derivative to give experimental proof of the formation of TICT state. This motivated us to provide a detailed description of the excited state processes in $N$-propyl-4-piperidinyl-1,8-naphthalimide (2, see Fig. 1), combining experimental and theoretical methods. $N$-Propyl-1,8naphthalimide (1) was used as reference compound. The rate coefficients of the reversible CT $\rightarrow$ TICT conversion were determined in different solvents at different temperatures, and were interpreted applying a kinetic barrier consisting of three terms, the inherent barrier of the reaction, and the contributions of the solvent viscosity and solvent polarity. The potential energy surfaces in the $S_{0}$ and $S_{1}$ states were calculated, along the coordinate of the turning motion which was conclusive concerning the direction of the twisting. The theoretical calculations confirmed a more efficient charge transfer of the dark TICT state compared to the CT state.

\section{Experimental section}

\section{General}

$\mathbf{1}$ and $\mathbf{2}$ were prepared as described elsewhere. ${ }^{27,28}$ Acetonitrile $(\mathrm{MeCN})$, propionitrile (EtCN), butyronitrile $(\mathrm{PrCN})$, valeronitrile (BuCN), caprylonitrile (HepCN), ethylene glycol (EG), methanol $(\mathrm{MeOH})$ and ethanol (EtOH) solvents were purchased from
Sigma Aldrich. MeCN was HPLC grade, $\mathrm{MeOH}$ and $\mathrm{EtOH}$ were spectroscopy grade and they were used without further purification. The other nitriles and the ethylene glycol were used after column chromatography purification using a mixed stationary phase (aluminium oxide 'Alumina B' and activated carbon) to remove the luminescent impurities.

\section{Spectroscopic experiments}

Absorption spectra were recorded using an Agilent 8453 diode array spectrometer. The steady state fluorescence spectra were measured on an Edinburgh Instruments FLS920 instrument, the recorded spectra were corrected with the characteristic of the light source and the detector. Time-resolved fluorescence experiments were performed using the frequency-doubled output of a Ti:sapphire laser system (Chameleon Ultra, Coherent) tuned to $820 \mathrm{~nm}$ and $700 \mathrm{~nm}$ which results in $410 \mathrm{~nm}$ and $350 \mathrm{~nm}$ excitation wavelengths for 2 and 1, respectively. A pulse picker was employed to reduce the repetition rate from 80 to $8 \mathrm{MHz}$. The fluorescence was detected using a monochromator (Newport CS260-02) coupled to a PMT (Hamamatsu). The overall instrument response function (IRF) was $\sim 20-25$ ps (fwhm). A thermostated sample holder was used for the temperature dependent experiments in EG and a liquid nitrogen cryostat was used for the measurements at $77 \mathrm{~K}$ in frozen solvent glass (EtOH: $\mathrm{MeOH}, 4: 1)$. All the experiments were performed at a concentration of $10 \mu \mathrm{M}$ in $1 \times 1 \mathrm{~cm}$ quartz cuvettes.

\section{Methods}

\section{Dipole moment change}

The dipole moment changes were determined from the absorption and fluorescence spectral data obtained in alkyl nitrils, using the Lippert-Mataga equation, ${ }^{29,30}$

$$
\Delta \tilde{\nu}=\tilde{\nu}_{\mathrm{a}}-\tilde{\nu}_{\mathrm{fl}}=\frac{2 \cdot \Delta f \cdot\left(\mu_{\mathrm{g}}-\mu_{\mathrm{e}}\right)^{2}}{4 \cdot \pi \cdot \varepsilon_{0} \cdot h \cdot c \cdot a_{0}{ }^{3}}+C
$$

where $\tilde{\nu}_{\mathrm{a}}$ and $\tilde{\nu}_{\mathrm{fl}}$ are the wavenumbers of absorption and fluorescence maxima, $\mu_{\mathrm{g}}$ and $\mu_{\mathrm{e}}$ are the dipole moments in the ground and excited states - $\mu_{\mathrm{g}}$ was calculated at the DFT level (for details see Computational section) $-\varepsilon_{0}$ is the vacuum permittivity, $h$ is the Planck constant, $c$ is the velocity of light, $a_{0}$ is the radius of the Onsager cavity. $a_{0}$ was also determined by quantum chemical calculations, it was taken as the sphere equivalent radius of the volume inside a contour of 0.001 electrons per Bohr ${ }^{3}$ density plus 0.5 Angström (this is the recommended radius for self consistent reaction field (SCRF) calculations). ${ }^{31} C$ in eqn (1) is a fitted constant, $\Delta f$ is the orientation polarizability,

$$
\Delta f=\frac{\varepsilon_{\mathrm{r}}-1}{2 \cdot \varepsilon_{\mathrm{r}}+1}-\frac{n^{2}-1}{2 \cdot n^{2}+1},
$$

where $\varepsilon_{\mathrm{r}}$ is the dielectric permittivity, $n$ is the refractive index of the medium. 


\section{Quantum yield determination}

The fluorescence quantum yields of NIs were determined using quinine sulfate (in $0.1 \mathrm{M} \mathrm{H}_{2} \mathrm{SO}_{4}$ ) as reference, applying the formula ${ }^{32}$

$$
\Phi_{\mathrm{NI}}=\Phi_{\text {ref }} \frac{\int I_{\mathrm{NI}}(\tilde{\nu}) \mathrm{d} \tilde{\nu}}{\int I_{\text {ref }}(\tilde{\nu}) \mathrm{d} \tilde{\nu}} \frac{n_{\text {org }}^{2}}{n_{\text {water }}^{2}} \frac{A_{\text {ref }}}{A_{\mathrm{NI}}}
$$

The quantum yield of quinine sulfate, $\Phi_{\text {ref }}$ is $0.53^{33}$ the subsequent fractionals in eqn (3) are the ratios of the integrated fluorescence intensities, the refractive indices and the absorbances, the latter at the excitation wavelength.

\section{Theoretical calculations}

Theoretical calculations were performed to investigate the TICT process and to calculate the Onsager radius of NIs using the Gaussian09 program package. ${ }^{31}$ The minimal-energy geometries were created in three steps. Firstly, we carried out a conformational analysis using the Merck molecular force field (MMFF94) with the Marvin Beans program package. ${ }^{34}$ Secondly, the geometries with up to $8 \mathrm{~kJ} \mathrm{~mol}^{-1}$ from the lowest energy conformer were optimized using PM6-D3H4 (parametrized model 6 with dispersion correction) implemented in MOPAC2016. ${ }^{35,36}$ Thereafter, the minimal energy conformer was optimized at the density functianol theory (DFT) level employing the PBE0 functional with the $6-311++G^{* *}$ basis set, Onsager radius and ground state dipole moments were calculated in vacuum. ${ }^{37,38}$

The geometries of 2 were optimized at fixed dihedral angles $(\Phi)$ (Fig. 1) for the investigation of the TICT process in vacuum using the $6-31++G^{* *}$ basis set with the same functional. The excited state energies were calculated the same way at the time-dependent DFT (TD-DFT) level of theory.

\section{Results and discussions}

\section{Solvatochromism in absorption and fluorescence spectra}

Compound $\mathbf{1}$ has a vibrational fine structure in its absorption as well as in its emission spectra. Besides, the absorption and emission spectra are nearly mirror images, which indicates that the geometry of the vibrationally relaxed $S_{1}$ state, from where the emission occurs, is close to the ground state structure. The Stokes shift of 1 is small $\left(3540 \mathrm{~cm}^{-1}\right)$, its value is similar as for the parent 1,8-naphthalimide. ${ }^{39}$

The excited state properties of $N$-propyl-1,8-naphthalimide are strongly modified by the piperidinyl substitution. As can be seen in Fig. 2, the longest wavelength absorption band of 1, appearing at $331 \mathrm{~nm}$ shifts strongly to the red, to $405 \mathrm{~nm}$ in the spectrum of 2 , indicating that the lowest energy excited state is stabilized due to a strong coupling of the electronic structures of the piperidinyl and 1,8-naphthalimide moieties. The electron donating piperidine substituent increases the difference between the $S_{0}$ and $S_{1}$ state energy levels: the fluorescence band located at $375 \mathrm{~nm}$ in the spectrum of 1 shifts to $535 \mathrm{~nm}$ in the spectrum of 2 . The corresponding Stokes shifts are $3540 \mathrm{~cm}^{-1}$ for 1 and $6000 \mathrm{~cm}^{-1}$ for compound 2 .

The solvatochromic behavior of the compounds was investigated in a homologous series of nitriles, minimizing the effect of the solvent specific interactions on the results. The spectral data of 1 and $\mathbf{2}$ in the nitrile solvents, and the dipole moment changes obtained from the Lippert-Mataga equation - eqn (1) are collected in Table 1, the Lippert-Mataga plot is shown in Fig. 3 These results demonstrate that $\mathbf{1}$ is not a charge transfer dye, whereas the radiative excited state of $\mathbf{2}$ has a moderate charge transfer character. The fluorescence quantum yield of 2 grows with the decrease of the polarity, suggesting that in addition to its moderately polar emissive CT state, 2 has a more polar, but dark state and the deactivation of the CT state via this dark state is more efficient in solvents of higher polarity.

\section{Effect of viscosity on fluorescence spectrum}

An indication for the TICT character of this dark state was provided by the fluorescence spectra of 2 in EG at different temperatures. As can be seen in Fig. 4, the fluorescence intensity strongly decreased at higher temperatures, i.e. at lower viscosities, but the shape of the spectra did not change. This can arise from
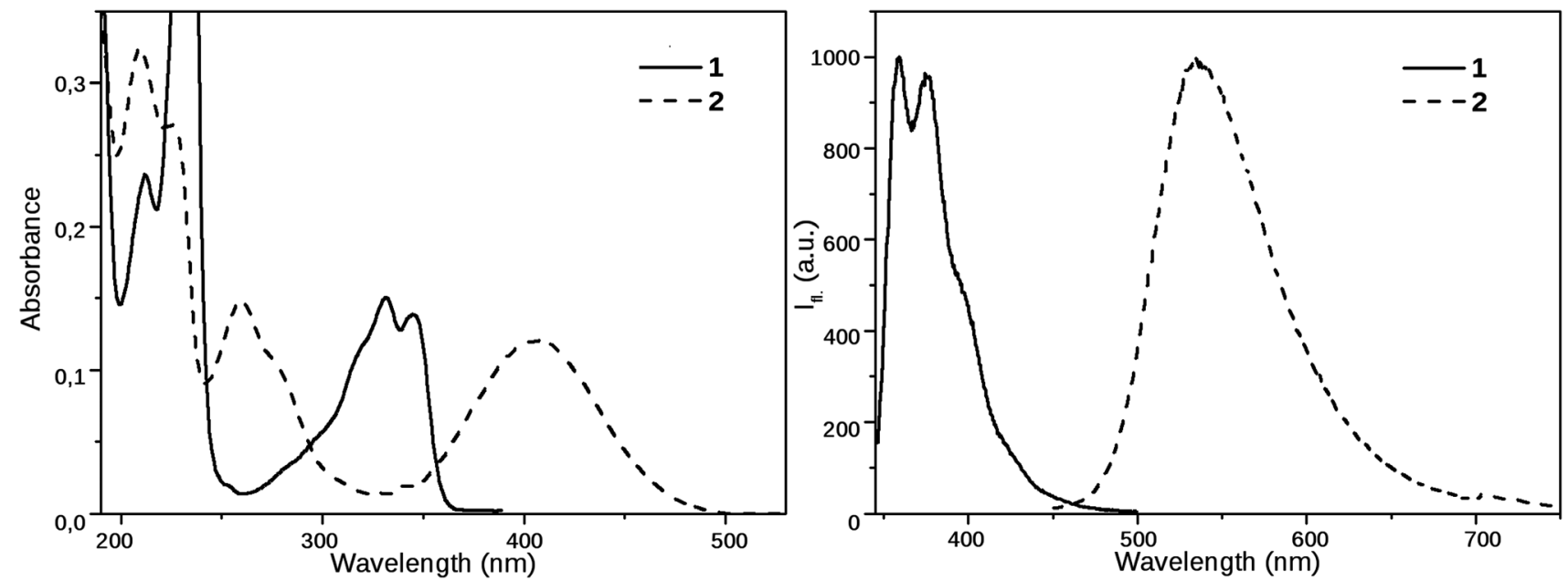

Fig. 2 Steady state absorption and emission spectra of $\mathbf{1}$ and $\mathbf{2}$ in MeCN (emission spectra were recorded using excitation at $330 \mathrm{~nm}$ and $405 \mathrm{~nm}$, respectively). 
Table 1 Spectral properties of $\mathbf{1}$ and $\mathbf{2}$ in the homologous series of nitriles, their Onsager radius, ground state dipole moments (calculated theoretically) and dipole moment changes obtained from the Lippert-Mataga analyses

\begin{tabular}{|c|c|c|c|c|c|c|c|c|c|}
\hline & & \multicolumn{5}{|l|}{ Solvents } & $a_{0}(\AA)$ & $\mu_{\mathrm{g}}(\mathrm{D})$ & $\Delta \mu$ (D) \\
\hline 1 & $\begin{array}{l}\lambda_{\text {abs }} / \lambda_{\mathrm{em}}(\mathrm{nm}) \\
\text { Fl. quant. yield }\end{array}$ & $\begin{array}{l}331 / 375 \\
0.023\end{array}$ & $\begin{array}{l}332 / 375 \\
0.017\end{array}$ & $\begin{array}{l}332 / 379 \\
0.013\end{array}$ & $\begin{array}{l}332 / 374 \\
0.012\end{array}$ & $\begin{array}{l}332 / 374 \\
0.015\end{array}$ & 4.65 & 3.91 & 5.35 \\
\hline 2 & $\begin{array}{l}\lambda_{\text {abs }} / \lambda_{\text {em }}(\mathrm{nm}) \\
\text { Fl. quant. yield }\end{array}$ & $\begin{array}{l}405 / 535 \\
0.043\end{array}$ & $\begin{array}{l}405 / 532 \\
0.069\end{array}$ & $\begin{array}{l}405 / 529 \\
0.11\end{array}$ & $\begin{array}{l}404 / 525 \\
0.17\end{array}$ & $\begin{array}{l}404 / 524 \\
0.32\end{array}$ & 5.74 & 7.52 & 10.4 \\
\hline
\end{tabular}

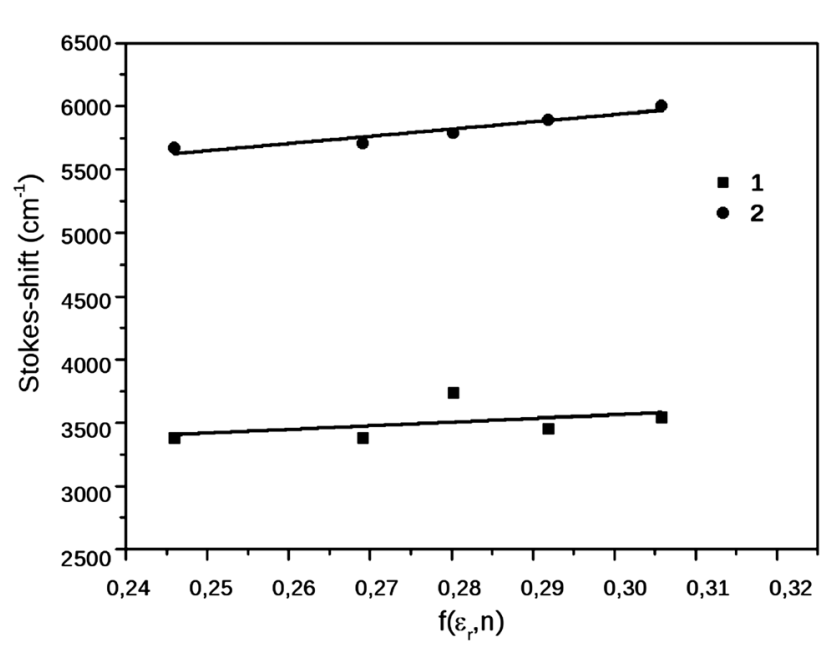

Fig. 3 Lippert-Mataga plot of $\mathbf{1}$ and $\mathbf{2}$ in the homologous series of nitriles

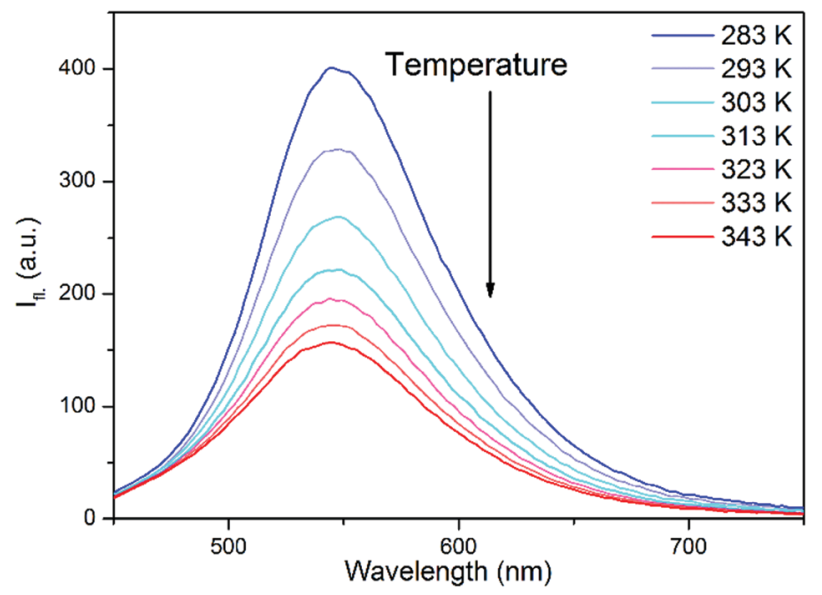

Fig. 4 Fluorescence spectra of $\mathbf{2}$ in EG at different temperatures. Excitation wavelength is $410 \mathrm{~nm}$.

a deactivation channel via a dark TICT species, and the twist of the piperidine moiety is favored at low viscosities. The fluorescence spectrum of $\mathbf{1}$ in EG (not shown here) was much less sensitive to the temperature.

\section{Fluorescence decay dynamics as functions of polarity and viscosity}

A further insight into the excited state processes in the two NIs were performed by time resolved fluorescence experiments. The effect of polarity on the excited state dynamics was
Table 2 Fitted amplitudes $(A)$ and fluorescence lifetimes $(\tau)$ in alkyl nitriles. Emission wavelengths are $370 \mathrm{~nm}$ (1) and $540 \mathrm{~nm}$ (2)

\begin{tabular}{|c|c|c|c|c|c|c|c|c|c|c|}
\hline \multicolumn{3}{|c|}{ MeCN } & \multicolumn{2}{|l|}{ EtCN } & \multicolumn{2}{|l|}{ PrCN } & \multicolumn{2}{|l|}{ BuCN } & \multicolumn{2}{|c|}{ HepCN } \\
\hline & $A(\%)$ & $\tau(\mathrm{ns})$ & $A(\%)$ & $\tau(\mathrm{ns})$ & $A(\%)$ & $\tau(\mathrm{ns})$ & $A(\%)$ & $\tau(\mathrm{ns})$ & $A(\%)$ & $\tau(\mathrm{ns})$ \\
\hline & 99.0 & 0.151 & 99.6 & 0.093 & 99.7 & 0.083 & 99.5 & 0.092 & 99.5 & 0.097 \\
\hline & 1.0 & 1.073 & 0.4 & 1.357 & 0.3 & 1.404 & 0.5 & 1.441 & 0.5 & 1.455 \\
\hline & 97.2 & 1.26 & 82.0 & 2.387 & 82.1 & 3.477 & 52.4 & 3.272 & 100.0 & 6.260 \\
\hline & 2.8 & 8.371 & 18.0 & 3.475 & 17.9 & 4.727 & 47.6 & 4.411 & & \\
\hline
\end{tabular}

investigated in the same series of nitriles as applied in the steady-state measurements. In addition, in case of 2 , the TICT character of the non-radiative decay was studied by measuring the fluorescence decay curves in ethylene glycol (EG), at different temperatures between 10 and $70{ }^{\circ} \mathrm{C}$, where the viscosity decreases from 34.8 to $4.0 \mathrm{cP}$, promoting the twist of the piperidine moiety. We note that the raise of temperature also has - a minor - opposite effect on the formation of the TICT state, as the polarity of EG decreases and that variation hinders the charge transfer process.

The piperidine substituted derivative 2 showed a biexponential decay in the nitrile solvents (Table 2), as well as in EG (Table 3), whereas the reference compound 1 showed biexponential decays in the nitriles and monoexponential decays

Table 3 Fitted amplitudes $(A)$ and fluorescence lifetimes $(\tau)$ in EG at different temperatures. Emission wavelengths are $370 \mathrm{~nm}$ (1) and $540 \mathrm{~nm}$ (2)

\begin{tabular}{|c|c|c|c|c|c|c|}
\hline \multirow{2}{*}{$\begin{array}{l}\text { Temperature } \\
\text { (K) }\end{array}$} & \multirow{2}{*}{$\begin{array}{l}\text { Viscosity }^{40} \\
\text { (cP) }\end{array}$} & \multirow{2}{*}{$\begin{array}{l}\text { Dielectric } \\
\text { constant }^{41}(-)\end{array}$} & \multicolumn{2}{|l|}{1} & \multicolumn{2}{|l|}{$\underline{2}$} \\
\hline & & & $A(\%)$ & $\tau(\mathrm{ns})$ & $A(\%)$ & $\tau(\mathrm{ns})$ \\
\hline \multirow[t]{2}{*}{283} & 34.8 & 43.5 & 100.0 & 1.329 & 0.8 & 6.508 \\
\hline & & & & & 99.2 & 0.143 \\
\hline \multirow[t]{2}{*}{293} & 22.0 & 41.3 & 100.0 & 1.218 & 1.1 & 6.477 \\
\hline & & & & & 98.9 & 0.107 \\
\hline \multirow[t]{2}{*}{303} & 14.5 & 39.3 & 100.0 & 1.057 & 0.8 & 6.446 \\
\hline & & & & & 99.2 & 0.083 \\
\hline \multirow[t]{2}{*}{313} & 10.0 & 37.3 & 100.0 & 0.944 & 0.8 & 6.355 \\
\hline & & & & & 99.2 & 0.071 \\
\hline \multirow[t]{2}{*}{323} & 7.2 & 35.4 & 100.0 & 0.844 & 0.7 & 6.315 \\
\hline & & & & & 99.3 & 0.060 \\
\hline \multirow[t]{2}{*}{333} & 5.3 & 33.6 & 100.0 & 0.750 & 0.8 & 6.260 \\
\hline & & & & & 99.2 & 0.056 \\
\hline \multirow[t]{2}{*}{343} & 4.0 & 31.9 & 100.0 & 0.680 & 0.9 & 6.233 \\
\hline & & & & & 99.1 & 0.052 \\
\hline
\end{tabular}




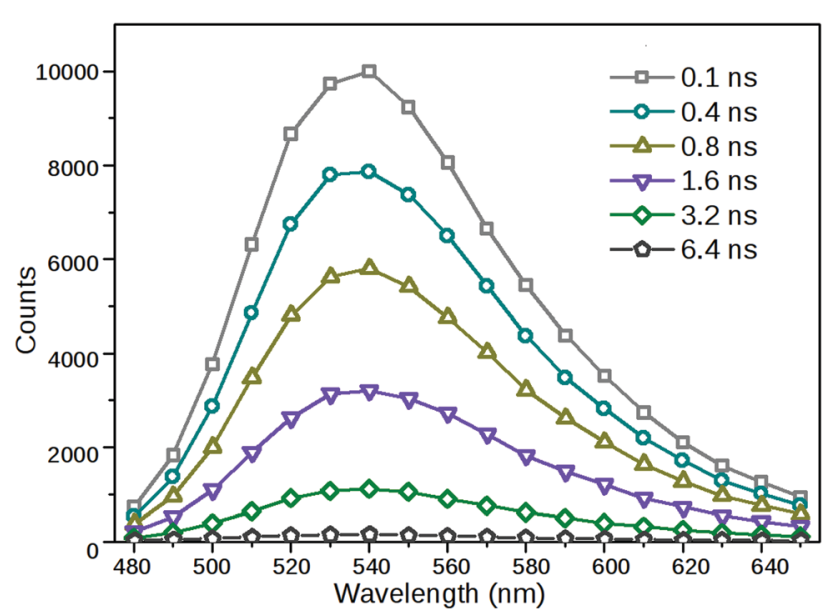

Fig. 5 Time-resolved fluorescence spectra of $\mathbf{2}$ in MeCN. Excitation wavelength is $410 \mathrm{~nm}$.

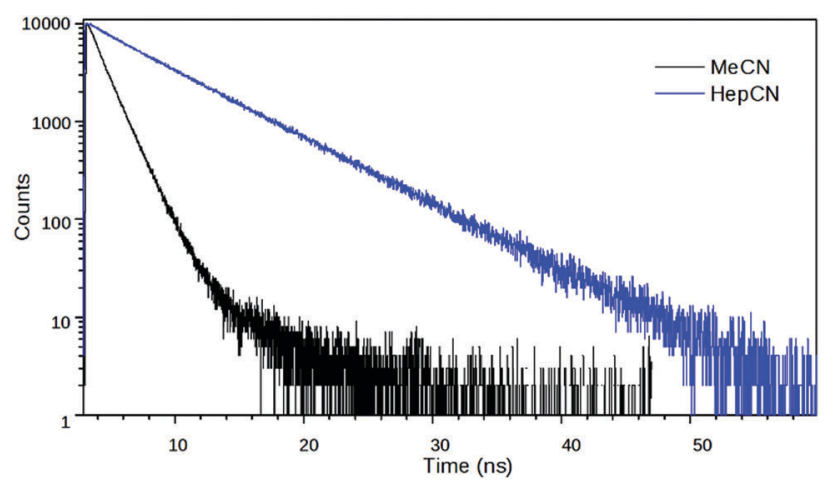

Fig. 6 Fluorescence decay curves of 2 in $\mathrm{MeCN}$ and $\mathrm{HepCN}$ at $540 \mathrm{~nm}$.

in EG. In the case of $\mathbf{2}$, the decay curves were recorded at five different wavelengths $(500,520,540,570,600 \mathrm{~nm})$ in all the solvents applied. The curves could be well fitted by the same time constants and relative amplitudes, which means that only a single radiative excited species had been detected.

As a further demonstration that a single radiative species had been formed via excitation, time-resolved fluorescence spectra of 2 were acquired from decay traces measured at different emission wavelengths. As can be seen in Fig. 5, the shapes and positions of the fluorescence bands obtained at different delay times were identical, only their relative intensities were different.

The fitted lifetime data in the nitriles show that the decay kinetics of $\mathbf{2}$ is affected more strongly by the solvent polarity than the kinetics of $\mathbf{1}$ (Table 2 and Fig. 6). The relative weights of the slow components in the decays increase with decreasing solvent polarity which is a further signal that the non-radiative state of 2 has a much stronger charge transfer character than the radiative one. Besides, the measurements in EG at different temperatures show that the excited state kinetics of 2 is influenced by the solvent viscosity. The fast component accelerates at higher temperatures, i.e. at lower viscosities, supporting the hypothesis that the non-radiative state is a TICT state (Fig. 7).

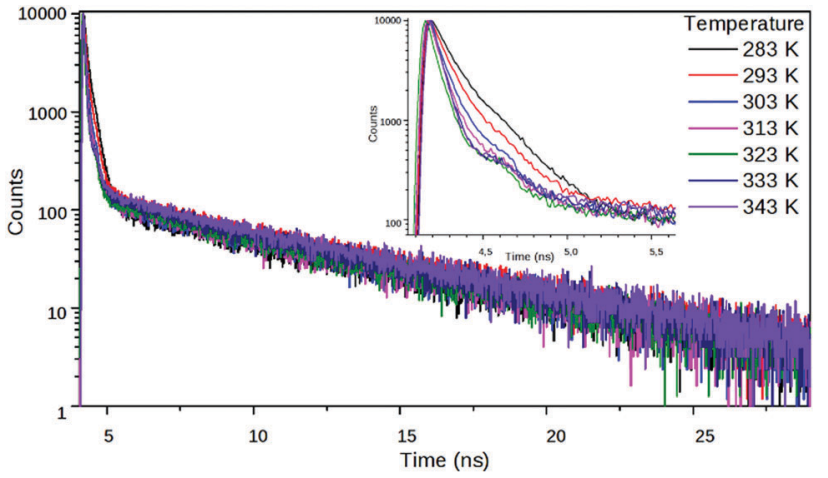

Fig. 7 Fluorescence decay curves of $\mathbf{2}$ in EG at different temperatures at $540 \mathrm{~nm}$.

\section{Kinetic analysis}

Our experimental observations on the piperidino derivative 2 are consistent with the kinetic model shown in Scheme 1.

The rates can be determined using the first order differential equation system

$$
\frac{\mathrm{d} \underline{c}(t)}{\mathrm{d} t}=\underline{\underline{K c}}(t)
$$

where $\underline{\underline{K}}$ is a matrix of the rate coefficients,

$$
\underline{\underline{K}}=\left(\begin{array}{cc}
-k_{x} & k_{-1} \\
k_{1} & -k_{y}
\end{array}\right),
$$

with

$$
\begin{gathered}
k_{x}=k_{\mathrm{C}}+k_{1} \\
k_{y}=k_{\mathrm{T}}+k_{-1},
\end{gathered}
$$

and $\underline{c}(t)$ is the vector of the concentrations as functions of time,

$$
\underline{c}(t)=\left(\begin{array}{l}
c_{\mathrm{C}}(t) \\
c_{\mathrm{T}}(t)
\end{array}\right)
$$

the indices $\mathrm{C}$ and $\mathrm{T}$ refer to the excited state species $\mathrm{CT}$ and TICT, respectively.

Integrating eqn (4) with respect to the time we get,

$$
\underline{c}(t)=\exp (\underline{\underline{K}} t) \underline{c_{0}}
$$

where $\underline{c_{0}}$ is the concentration vector at $t=0$.

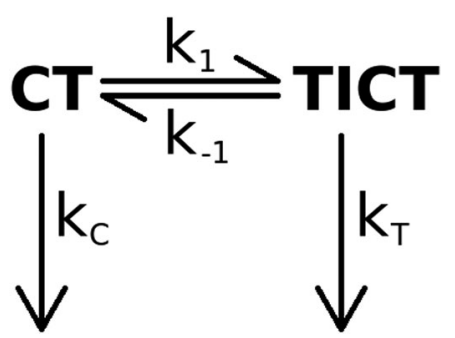

Scheme 1 The proposed kinetic scheme for the excited state processes of 2 . 
The fluorescence lifetimes, $\tau_{1}$ and $\tau_{2}$ are the negative reciprocals of the eigenvalues of the $\underline{K}$ matrix, $\lambda_{1}$ and $\lambda_{2}$,

$$
-\frac{1}{\tau_{1,2}}=\lambda_{1,2}=\frac{-k_{x}-k_{y} \mp \sqrt{4 k_{1} k_{-1}+k_{x}^{2}-2 k_{x} k_{y}+k_{y}^{2}}}{2}
$$

The integrated forms of the rate equations are ${ }^{42}$

$$
\begin{aligned}
c_{\mathrm{C}}(t) & =\left(\frac{-k_{x}-\lambda_{2}}{\lambda_{1}-\lambda_{2}} \mathrm{e}^{\lambda_{1} t}+\frac{k_{x}+\lambda_{1}}{\lambda_{1}-\lambda_{2}} \mathrm{e}^{\lambda_{2} t}\right) c_{0, \mathrm{C}} \\
& =\left(a_{\mathrm{C}, 1} \mathrm{e}^{-t / \tau_{1}}+a_{\mathrm{C}, 2} \mathrm{e}^{-t / \tau_{2}}\right) c_{0, \mathrm{C}}
\end{aligned}
$$

and

$$
\begin{aligned}
c_{\mathrm{T}}(t) & =\left(\frac{k_{1}}{\lambda_{1}-\lambda_{2}} \mathrm{e}^{\lambda_{1} t}-\frac{k_{1}}{\lambda_{1}-\lambda_{2}} \mathrm{e}^{\lambda_{2} t}\right) c_{0, \mathrm{C}} \\
& =a_{\mathrm{T}}\left(\mathrm{e}^{-t / \tau_{1}}-\mathrm{e}^{-t / \tau_{2}}\right) c_{0, \mathrm{C}}
\end{aligned}
$$

which can be obtained expanding eqn (7) by calculating the matrix exponential and assuming that $c_{0, \mathrm{~T}}=0$.

The TICT species is non-fluorescent in the present case. The time constants $\tau_{1}$ and $\tau_{2}$ in eqn (9) are provided by the fitting of the decay curves to biexponential functions, the ratio of $a_{\mathrm{c}, 1}$ and $a_{\mathrm{c}, 2}$ can be obtained as the ratio of the relative amplitudes of the two decay components (pairs of A values in Tables 2 and 3),

$$
\frac{a_{\mathrm{C}, 1}}{a_{\mathrm{C}, 2}}=\frac{-k_{x}-\lambda_{2}}{k_{x}+\lambda_{1}}=\frac{A_{\mathrm{C}, 1}}{A_{\mathrm{C}, 2}} .
$$

Of the four rate coefficients in Scheme $1, k_{\mathrm{C}}$ can be determined directly, measuring the fluorescence decay of the CT species in a frozen solvent glass.

$k_{x}$ can be calculated by eqn (11), from the time constants $\tau_{1}\left(=-1 / \lambda_{1}\right), \tau_{2}\left(=-1 / \lambda_{2}\right)$ and amplitudes of the biexponential decay curves. Using that

$$
\lambda_{1}+\lambda_{2}=-k_{x}-k_{y}
$$

$k_{y}$ can be calculated as well. In the possession of the values of $k_{\mathrm{C}}$ and $k_{x}, k_{1}$ can be obtained from eqn (5). Thereafter $k_{-1}$ can be calculated, expressing it from eqn (8),

$$
\begin{aligned}
k_{-1} & =\frac{k_{x} k_{y}+k_{x} \lambda_{1}+k_{y} \lambda_{1}+\lambda_{1}^{2}}{k_{1}} \\
& =\frac{k_{x} k_{y}+k_{x} \lambda_{2}+k_{y} \lambda_{2}+\lambda_{2}^{2}}{k_{1}}
\end{aligned}
$$

Finally $k_{\mathrm{T}}$ can be calculated from eqn (6).

In a $4: 1 \mathrm{EtOH}: \mathrm{MeOH}$ solvent mixture, 2 had a biexponential decay - with time constants (relative amplitudes) of $7.79 \mathrm{~ns}$ (1.4) and $0.188 \mathrm{~ns}$ (98.6) - whereas it had a monoexponential decay with a time constant of $9.68 \mathrm{~ns}$ at $77 \mathrm{~K}$ in frozen solvent glass where the turning of the piperidine unit is hampered so the TICT state cannot form (Fig. 8). The reciprocal of the latter lifetime was accepted as the value of $k_{\mathrm{C}}$. Subsequently, $k_{1}, k_{-1}$ and $k_{\mathrm{T}}$ were calculated from the time constants and amplitudes, following the method described above.

As can be seen in Tables 4 and $5, k_{1}$ decreases with the decreasing solvent polarity and with increasing viscosity as is expected for a TICT state formation. Moreover, the $k_{\mathrm{T}}$ rate

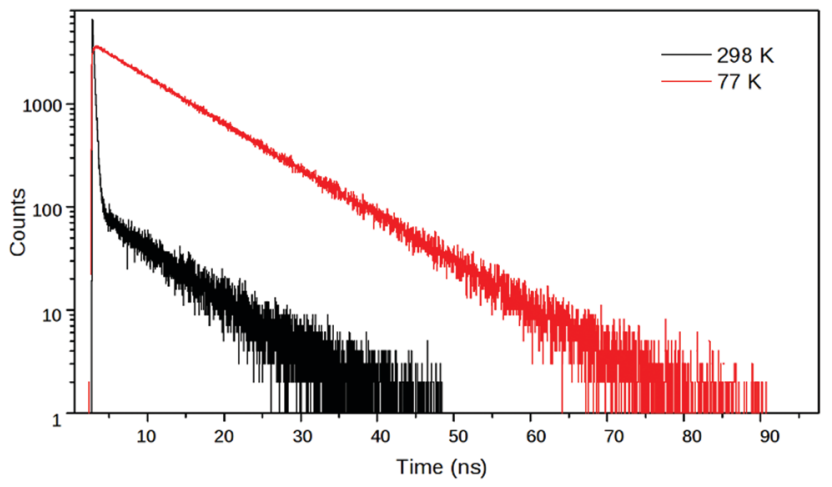

Fig. 8 Fluorescence decay curves of 2 at $\lambda_{\mathrm{em}}=540 \mathrm{~nm}$ in $\mathrm{EtOH}: \mathrm{MeOH}$ $(4: 1)$ at room temperature and at $77 \mathrm{~K}$ in frozen solvent glass.

Table 4 Rate constants for the excited state pathways of $\mathbf{2}$ in EG

\begin{tabular}{llll}
\hline Temperature $(\mathrm{K})$ & $k_{1}\left(\mathrm{~ns}^{-1}\right)$ & $k_{-1}\left(\mathrm{~ns}^{-1}\right)$ & $k_{\mathrm{T}}\left(\mathrm{ns}^{-1}\right)$ \\
\hline 283 & 6.8 & 0.053 & 0.15 \\
293 & 9.2 & 0.10 & 0.15 \\
303 & 12 & 0.091 & 0.16 \\
313 & 14 & 0.11 & 0.16 \\
323 & 16 & 0.12 & 0.16 \\
333 & 18 & 0.14 & 0.16 \\
343 & 19 & 0.16 & 0.16
\end{tabular}

coefficient shows only a minor sensitivity to the solvent polarity and is insensitive to the solvent viscosity.

The simple Arrhenius plot of the $k_{1}-s$, the rate coefficients of the CT $\rightarrow$ TICT process obtained in EG, shown in Fig. 9, is nonlinear, suggesting that the effect of the viscosity should be taken in account. The ratio of $k_{1} / k_{-1}$, which can be considered the equilibrium constant of the excited state reaction, did not change significantly, which can be because the dielectric constant of EG changed only from 44 to 32 .

We can rule out that the non-linearity of the Arrhenius plot is related to solvent relaxation processes since the longitudinal relaxation time constant of EG at room temperature is around $10 \mathrm{ps},{ }^{43,44}$ whereas the $k_{1}$ rate coefficient at $293 \mathrm{~K}\left(9.2 \mathrm{~ns}^{-1}\right)$ corresponds to a CT $\rightarrow$ TICT conversion slower by one order of magnitude. The effects of the viscosity and polarity on the reaction rate were taken into account by taking the activation

\begin{tabular}{|c|c|c|c|c|c|}
\hline & & \multicolumn{4}{|c|}{ Solvents } \\
\hline & & $\mathrm{MeCN}$ & EtCN & PrCN & BuCN \\
\hline \multirow[t]{3}{*}{2} & $k_{1}\left(\mathrm{~ns}^{-1}\right)$ & 0.671 & 0.292 & 0.171 & 0.165 \\
\hline & $k_{-1}\left(\mathrm{~ns}^{-1}\right)$ & 0.018 & 0.009 & 0.005 & 0.009 \\
\hline & $k_{\mathrm{T}}\left(\mathrm{ns}^{-1}\right)$ & 0.120 & 0.303 & 0.220 & 0.255 \\
\hline
\end{tabular}
energy as the sum of three contributions, ${ }^{45}$

$$
E_{\mathrm{a}} \approx E_{0}+E_{\mathrm{visc}}+E_{\mathrm{pol}}
$$

where $E_{0}$ is the intrinsic barrier, $E_{\text {visc }}$ is the effect of viscosity and $E_{\mathrm{pol}}$ is the effect of the polarity on the Arrhenius type barrier. Thus, the non-linearity of the Arrhenius plot can

Table 5 Rate constants for the excited state pathways of $\mathbf{2}$ in the series of nitriles 


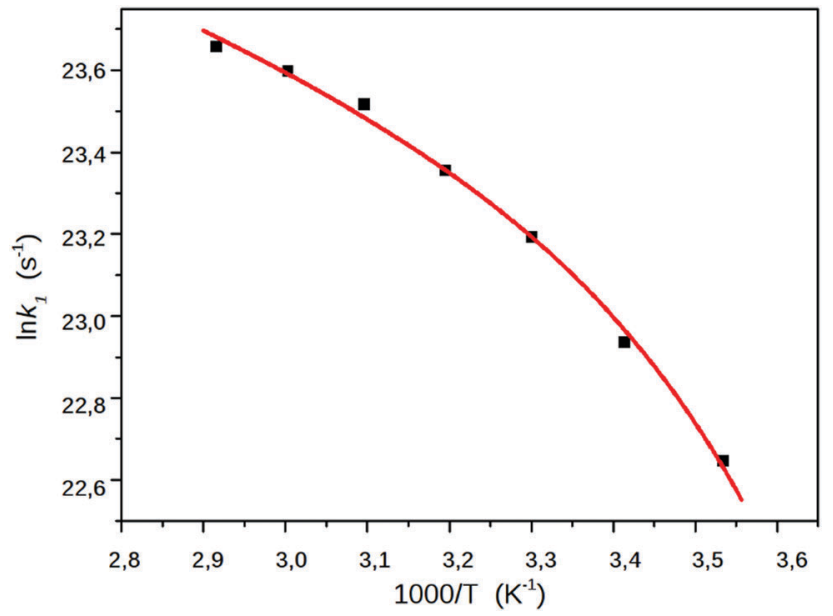

Fig. 9 The Arrhenius plot of $k_{1}$, the rate coefficient of the CT $\rightarrow$ TICT process in EG. The squares denote the experimentally determined $k_{1}$ values, the red line represents a least square fitting to eqn (19).

originate from the temperature dependency of the second two contributions in eqn (14).

The term $E_{\text {visc }}$ was assumed as the energy of a disk rotating in a viscous fluid, ${ }^{46}$

$$
E_{\mathrm{visc}}(T) \approx \eta(T) b=2 \pi \eta(T) \int_{0}^{R} r^{2} \frac{\partial v}{\partial z} \Delta \Phi \mathrm{d} r
$$

where $\eta$ is the dynamic viscosity, $T$ is the temperature, the integral multiplied by $2 \pi$ was considered constant and denoted by $b$. The radius of the disc (piperidinyl group) is denoted with $r, v$ is a velocity component, $z$ is a polar coordinate (the axis of rotation) and $\Phi$ is the defined dihedral angle (see Fig. 1). $E_{\text {visc }}$ changes rapidly in the temperature range of the kinetic experiments: its value at $343 \mathrm{~K}$ is only $11 \%$ of the value at $283 \mathrm{~K}$.

The third term in eqn (14) was considered as the solvation energy excess of 2 at the transition state (TS) in the CT-TICT reaction,

$$
E_{\mathrm{pol}}(T)=E_{\mathrm{solv}, \mathrm{TS}}(T)-E_{\mathrm{solv}, \mathrm{CT}}(T)
$$

The solvation energies were estimated using the Onsagermodel, according which the energy of a solute with a dipole, $\mu$, interacts with the Onsager reaction field, $R$, in a spherical cavity, ${ }^{47}$

$$
E_{\mathrm{solv}}=-\frac{1}{2} \mu R,
$$

where the reaction field is calculated as

$$
R=\frac{2[\varepsilon(T)-1] \mu}{[2 \varepsilon(T)+1] a^{3}} .
$$

$\varepsilon$ in eqn (18) denotes the dielectric constant and $a$ is the Onsager radius.

The dipole moments of the CT and the TS species were calculated at the TD-DFT level (Fig. $10,45^{\circ}$ and $-5^{\circ}$, respectively). The value of $E_{\mathrm{pol}}$ was calculated with these dipole moments at the lowest and highest temperatures applied
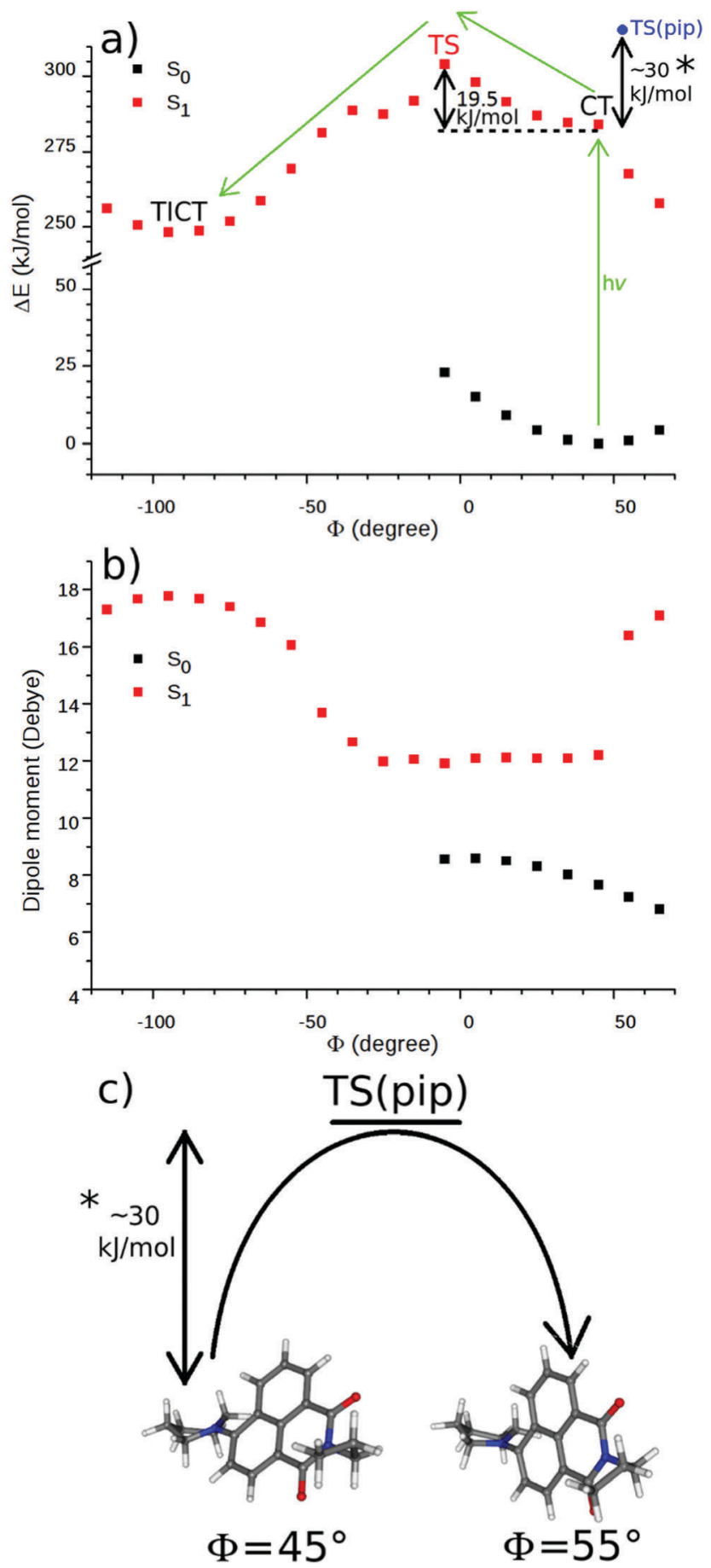

Fig. 10 Theoretical results of $\mathbf{2}$ calculated at DFT/TD-DFT level along the dihedral angle $-\Phi$ - in vacuum, (a) potential curve (the proposed reaction path is denoted with green arrows), (b) dipole moments, (c) the boat $\rightarrow$ chair transformation of the piperidine unit.

in the experiments ( 283 and $343 \mathrm{~K}$ ) and only a difference of $\sim 0.2 \mathrm{~kJ} \mathrm{~mol}^{-1}$ was obtained. Therefore, the temperature dependence of this term was neglected and the Arrhenius equation was written in the

$$
\ln k=\frac{-E_{0}-E_{\mathrm{pol}}}{R T}+\frac{-\eta(T) b}{R T}+\ln A
$$


form, where $R$ is the gas constant and $b$ (in $\mathrm{J}(\mathrm{cP} \mathrm{mol})^{-1}$ ) depends on the shape of the turning unit and also on the angle and angular velocity of the rotation.

As can be seen in Fig. 9, a nice fitting can be achieved by introducing the viscosity dependence in the kinetic barrier with the use of the expression (19). The values of the parameters of the function shown in the graph are $\ln A=26.07, E_{0}+E_{\mathrm{pol}}=$ $6.64 \mathrm{~kJ} \mathrm{~mol}^{-1}$ and $b=0.0415 \mathrm{~kJ}(\mathrm{cP} \mathrm{mol})^{-1}$.

\section{Quantum chemical calculations}

The theoretical calculations were performed at the DFT and the TD-DFT levels to explore the mechanism of the TICT formation of 2 further. The S1 state optimization starting from the ground state structure resulted in a similar structure with only a minor change of the dihedral angle (from $46.1^{\circ}$ to $43.7^{\circ}$ ) and with an increase of the dipole moment from 7.6 D to $12.2 \mathrm{D}$. The latter value is close to the experimental dipole moment of $N$-butyl-4butylamino-1,8-naphthalimide in excited state. ${ }^{16}$ This structure represents the CT state of 2 , the existence of which was proved experimentally by the solvatochromism of this naphthalimide derivative, shown in nitriles of different polarities.

The potential curves were calculated in vacuum, along the dihedral angle between the piperidinyl moiety and the naphthalimide ring system at the DFT/TD-DFT level. $\Phi$ was changed by $10^{\circ}$ in each step. The TICT state - the minimum on the $\mathrm{S}_{1}$ surface - was identified at a $\Phi$ of $-88^{\circ}$ with a dipole moment of $17.7 \mathrm{D}$ (see Fig. 10a and b). Its dark state property was confirmed by the TD-DFT calculation which resulted in a zero oscillator strength for the corresponding $\mathrm{S}_{1} \rightarrow \mathrm{S}_{0}$ vertical transition. In contrast, the CT state proved emissive also by the calculations, an oscillator strength of 0.224 was obtained for the vertical $S_{1} \rightarrow S_{0}$ deexcitation from the relaxed CT state.

As can be seen in Fig. 10a, the energy falls monotonously in the direction of the larger $\Phi$ angles (for the direction of the rotation see Fig. 1), which may suggest a barrierless process. A comparison of the optimized structures with fixed dihedral angles, however, reveals that there is a chair $\rightarrow$ boat conformational change of the piperidinyl unit at $\Phi=55^{\circ}$ (Fig. 10c). Probably, this arises from the repulsion of the $\mathrm{H}$ atoms at the bond between the piperidinyl and 1,8-naphthalimide moieties. A similar excited state folding motion of the piperidinyl group was investigated in detail experimentally in the case of some piperidinyl electron donor-other acceptor systems by Verhoeven et al. and barriers between 29 and $33 \mathrm{~kJ} \mathrm{~mol}^{-1}$ were obtained. ${ }^{48}$ In the direction of smaller $\Phi$ angles a barrier of $19.5 \mathrm{~kJ} \mathrm{~mol}^{-1}$ was obtained and the chair conformation of the piperidine ring was retained, suggesting that the turning is more probable in this direction. The dipole moment (see Fig. 10b) did not change significantly on the $S_{1}$ surface until $\Phi$ reached $-35^{\circ}$ then it increased rapidly to its maximum in the TICT state where three bonds around the piperidine $\mathrm{N}$ atom are coplanar.

Natural bond orbital analysis were performed on the stationary points of the $S_{1}$ surface (corresponding to the CT and the TICT structures) to investigate the changes in the electronic structures. In the CT state, the orbitals of the

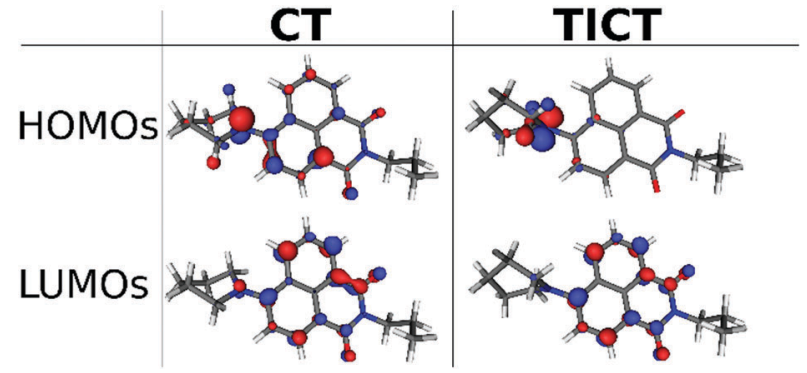

Fig. 11 The calculated molecular orbitals of the stationary points on the $\mathrm{S}_{1}$ surface at TD-DFT level.

piperidine nitrogen which form the bond with the piperidine carbons, are in $\mathrm{sp}^{2.16}$ and $\mathrm{sp}^{2.17}$ hybridization states and the orbital forming the bond with the naphthalimide carbon is in $\mathrm{sp}^{1.78}$ hybridization state. In the TICT state, the bonding orbitals are purely in $\mathrm{sp}^{2}$ hybridization state $\left(\mathrm{sp}^{1.98}, \mathrm{sp}^{2.02}\right.$ and $\mathrm{sp}^{2.00}$, respectively) as a result of the planar structure. The lone pairs are in $p(>99 \%)$ hybridization state in both cases.

It is instructive to inspect the shapes of the frontier molecular orbitals (MOs) (Fig. 11). The highest occupied MO (HOMO) of the CT state is located on the nitrogen and on the aromatic naphthalimide unit in similar amounts, the LUMO primarily on the aromatic system. In the TICT state the HOMO is purely on the piperidinyl unit, the LUMO is localized mainly on the aromatic system. This result clearly demonstrates that the charge transfer is more pronounced in the TICT state which has a higher dipole moment since the excited states correspond to a completely ( $>97 \%$ in both cases) HOMO $\rightarrow$ LUMO excitations.

\section{Conclusions}

In this paper, an experimental proof was presented for the formation of the TICT state in a 1,8-naphthalimide based pushpull system. The fluorescence decay data were analyzed using a two state system kinetic scheme. The viscosity and polarity dependent rate constants support the hypothesis that the TICT state formation is the main quenching process of the radiative excited state, CT, of 4-piperidinyl-1,8-naphthalimide. The monoexponential fluorescence decay in frozen solvent glass is a further evidence. A modified Arrhenius equation was employed on the excited state kinetic data in ethylene glycol which resulted in a nice fitting and in this way, we could extract the intrinsic activation energy of the TICT state formation.

The theoretical calculations show that the TICT species has a much stronger charge transfer character compared to the former CT state. Moreover, the results suggest that the turning is more likely in the direction of smaller $\Phi$ dihedral angles because there is a higher barrier of a conformational change in the other direction. Probably it is because of the repulsion of the $\mathrm{H}$ atoms at the bond between the piperidinyl and 1,8-naphthalimide moieties. The calculations confirm that the TICT state is dark and has a higher dipole moment compared the CT state. We hope that our results will help the development of novel 1,8-naphthalimide based molecular switches/probes and the investigation of their TICT states. 


\section{Conflicts of interest}

There are no conflicts to declare.

\section{Acknowledgements}

Financial support for this research was provided by Hungarian NKFIH (grant K 108752), LASERLAB-EUROPE (grant agreement no. 654148, European Union's Horizon 2020 research and innovation programme), European Union MSCA-ITN-2015-ETN Action program, ISPIC, under grant no. 675743 and Netherlands Organisation for Scientific Research in the framework of the Fund New Chemical Innovation (2015) TA under grant no. 731.015.206. The computing time granted on the Hungarian HPC Infrastructure at NIIF Institute, Hungary, is gratefully acknowledged.

\section{References}

1 A. P. de Silva, H. Q. N. Gunaratne, T. Gunnlaugsson, A. J. M. Huxley, C. P. McCoy, J. T. Rademacher and T. E. Rice, Chem. Rev., 1997, 97, 1515-1566.

2 R. A. Bissell, A. P. de Silva, H. Q. N. Gunarane, P. L. M. Lynch, G. E. M. Maguire, C. P. McCoy and K. R. A. S. Sandanayake, Top. Curr. Chem., 1993, 168, 223-264.

3 G. R. C. Hamilton, S. K. Sahoo, S. Kamila, N. Singh, N. Kaur, B. W. Hyland and J. F. Callan, Chem. Soc. Rev., 2015, 44, 4415-4432.

4 A. P. de Silva, H. Q. N. Gunaratne and C. P. McCoy, Nature, 1993, 364, 42-44.

5 R. M. Duke, E. B. Veale, F. M. Pfeffer, P. E. Kruger. and T. Gunnlaugsson, Chem. Soc. Rev., 2010, 39, 3936-3953.

6 D. Wang, X. Zhang, C. He and C. Duan, Org. Biomol. Chem., 2010, 8, 2923-2925.

7 J. B. Czirok, M. Bojtár, D. Hessz, P. Baranyai, L. Drahos and M. Kubinyi, I. Bitter, Sens. Actuators, B, 2013, 182, 280-287.

8 G. S. Loving and B. Imperiali, J. Am. Chem. Soc., 2008, 130, 13630-13638.

9 G. S. Loving, M. Sainlos and B. Imperiali, Trends Biotechnol., 2009, 28, 73-83.

10 V. Wintgens, P. Valat, J. Kossanyi, L. Biczok, A. Demeter and T. Bérces, J. Chem. Soc., Faraday Trans., 1994, 90, 411-421.

11 V. Wintgens, P. Valat, J. Kossanyi, A. Demeter, L. Biczok and T. Bérces, New J. Chem., 1996, 20, 1149-1158.

12 E. Martín, J. L. G. Coronado, J. J. Camacho and A. Pardo, J. Photochem. Photobiol., A, 2005, 175, 1-7.

13 L. Biczók, P. Valat and V. Wintgens, Phys. Chem. Chem. Phys., 1999, 1, 4759-4766.

14 S. Saha and A. Samanta, J. Phys. Chem. A, 2002, 106, 4763-4771.

15 A. Pardo, J. M. L. Poyato, E. Martin, J. J. Camacho, D. Reyman, M. F. Brana and J. M. Castellano, J. Photochem. Photobiol., A, 1989, 46, 323-328.

16 A. P. de Silva, H. Q. N. Gunaratne, J. L. Habib-Jiwan, C. P. McCoy, T. E. Rice and J. P. Soumillion, Angew. Chem., Int. Ed., 1995, 34, 1728-1731.
17 X. T. Jia, Y. Yang, Y. F. Xu and X. H. Qian, Pure Appl. Chem., 2014, 86, 1237-1246.

18 S. Zheng, P. L. M. Lynch, T. E. Rice, T. S. Moody, H. Q. N. Gunaratne and A. P. de Silva, Photochem. Photobiol. Sci., 2012, 11, 1675-1681.

19 P. A. Panchenko, A. N. Arkhipova, O. A. Fedorova, Y. V. Fedorov, M. A. Zakharko, D. E. Arkhipova and G. Jonusauskas, Phys. Chem. Chem. Phys., 2017, 19, 1244-1256.

20 Y. Luo, Y. Wang, S. Chen, N. Wang, Y. Qi, X. Zhang, M. Yang, Y. Huang, M. Li, J. Yu, D. Luo and Z. Lu, Small, 2017, 13, e1604113.

21 F. Y. Y. Wang, W. Zhu, Y. Huang, M.-H. Yang, H. Ai and Z.-Y. Lu, RSC Adv., 2014, 4, 36849-36853.

22 T. Liu, X. Liu, D. R. Spring, X. Qian, J. Cui and Z. Xu, Sci. Rep., 2014, 4, e5418.

23 Y. Sun, X. H. Liang, J. Fan and Q. Han, J. Lumin., 2013, 141, 93-98.

24 Y. Qian, M.-M. Cai, L.-H. Xie, G.-Q. Yang, S.-K. Wu and W. Huang, ChemPhysChem, 2011, 12, 397-404.

25 Y. Qian, M.-M. Cai, X.-H. Zhou, Z.-Q. Gao, X.-P. Wang, Y.-Z. Zhao, X.-H. Yan, W. Wei, L.-H. Xie and W. Huang, J. Phys. Chem. C, 2012, 116, 12187-12195.

26 J.-W. Li, Y. Qian, L.-H. Xie, Y.-P. Yi, W.-W. Li and W. Huang, J. Phys. Chem. C, 2015, 119, 2133-2141.

27 M. A. Landey-Alvarez, A. Ochoa-Teran, G. Pina-Luis, M. Martinez-Quiroz, M. Aguilar-Martiínez, J. Elias-Garcia, V. Miranda-Soto, J.-Z. Ramirez, L. Machi-Lara, V. L. Galvan and M. Ordonez, Supramol. Chem., 2016, 28, 892-906.

28 A. T. Peters and M. J. Bide, Dyes Pigm., 1985, 6, 349-375.

29 E. Lippert, Z. Elektrochem., 1957, 61, 962-975.

30 N. Mataga, Y. Kaifu and M. Koizumi, Bull. Chem. Soc. Jpn., 1956, 29, 465-470.

31 M. J. Frisch, G. W. Trucks, H. B. Schlegel, G. E. Scuseria, M. A. Robb, J. R. Cheeseman, G. Scalmani, V. Barone, B. Mennucci, G. A. Petersson, H. Nakatsuji, M. Caricato, X. Li, H. P. Hratchian, A. F. Izmaylov, J. Bloino, G. Zheng, J. L. Sonnenberg, M. Hada, M. Ehara, K. Toyota, R. Fukuda, J. Hasegawa, M. Ishida, T. Nakajima, Y. Honda, O. Kitao, H. Nakai, T. Vreven, J. A. Montgomery, Jr., J. E. Peralta, F. Ogliaro, M. Bearpark, J. J. Heyd, E. Brothers, K. N. Kudin, V. N. Staroverov, R. Kobayashi, J. Normand, K. Raghavachari, A. Rendell, J. C. Burant, S. S. Iyengar, J. Tomasi, M. Cossi, N. Rega, J. M. Millam, M. Klene, J. E. Knox, J. B. Cross, V. Bakken, C. Adamo, J. Jaramillo, R. Gomperts, R. E. Stratmann, O. Yazyev, A. J. Austin, R. Cammi, C. Pomelli, J. W. Ochterski, R. L. Martin, K. Morokuma, V. G. Zakrzewski, G. A. Voth, P. Salvador, J. J. Dannenberg, S. Dapprich, A. D. Daniels, Ö. Farkas, J. B. Foresman, J. V. Ortiz, J. Cioslowski and D. J. Fox, Gaussian 09, Revision $C$, Gaussian, Inc., Wallingford CT, 2009.

32 J. B. Birks, J. Res. Natl. Bur. Stand., Sect. A, 1976, 80, 389-399. 33 M. J. Adams, J. G. Highfield and G. F. Kirkbright, Anal. Chem., 1977, 49, 1850-1852.

34 MarvinSketch 16.4.25, ChemAxon, 2016, http://chemaxon.com. 35 J. J. P. Stewart, MOPAC2016, Stewart Computational Chemistry, Colorado Springs, CO, USA, 2016, http://OpenMOPAC.net. 
36 J. Řezáč and P. Hobza, J. Chem. Theory Comput., 2012, 8, 141-151. 37 J. P. Perdew, K. Burke and M. Ernzerhof, Phys. Rev. Lett., 1996, 77, 3865-3868.

38 C. Adamo and V. Barone, J. Chem. Phys., 1999, 110, 6158-6169.

39 A. Pardo, J. M. L. Poyato and E. Martin, J. Photochem., 1987, 36, 323-329.

40 A. K. Coker, Ludwig's Applied Process Design for Chemical and Petrochemical Plants, Elsevier, 4th edn, 2015.

41 D. R. Lide, Handbook of chemistry and physics, 84th edn, CRC Press, 2003.

42 R. C. Evans, P. Douglas and H. D. Burrow, Applied Photochemistry, Springer, 2013.
43 M. Hecht and W. R. Fawcett, J. Phys. Chem., 1996, 100, 14248-14255.

44 A. Declémy, C. Rulliére and P. Kottis, Laser Chem., 1990, 10, 413-429.

45 Z. R. Grabowski, K. Rotkiewicz and W. Rettig, Chem. Rev., 2003, 103, 3899-4031.

46 L. T. Watson and C. Y. Wang, Phys. Fluids, 1979, 22, 2267-2269.

47 R. O. Watts, Mol. Phys., 1974, 28, 1069-1083.

48 B. Bleisteiner, T. Marian, S. Schneider, A. M. Brouwer and J. W. Verhoeven, Phys. Chem. Chem. Phys., 2001, 3, 5383-5392. 